\title{
EAl Endorsed Transactions

\section{An Energy Efficient Scheme using Mobility Prediction for Localization of Wireless Sensor Nodes}

\author{
D.N.Chouhan ${ }^{1, *}$ and T.K. Dubey ${ }^{2}$ \\ ${ }^{1}$ Research Scholar, Manipal University Jaipur, India \\ ${ }^{2}$ Associate Professor, Manipal University Jaipur, India
}

\begin{abstract}
Location estimation of sensor nodes in Wireless Sensor Networks is very essentials because without information of location the information is meaningless. Most of the range-free algorithms have low localization accuracy, low cost, and applications limited to indoor uses only. This paper proposes a Mobility Prediction localization algorithm using the Link expiration time estimation method, this concept brings continuous link among the anchors and the mobile sensor nodes. This gives more accurate position estimation (3.2\% of R) and employs fewer samples (average 20.58 per slot) for the task, hence results in less energy consumption than a Sequential Monte Carlo localization scheme. Both the algorithms are studied, analyzed and compared with speed of the mobile nodes in terms of localization error $(6.38 \%$ to $6.55 \%$ better on different anchor density) communication cost $(51.72 \%$ high), the number of samples taken per slot (average $58.8 \%$ less) and residual energy profile.
\end{abstract}

Keywords: WSNs, MCL, SMCL, Mobility prediction, Localization, Sensor nodes

Received on 28 February 2019, accepted on 09 April 2019, published on 26 April 2019

Copyright (C) 2019 D.N.Chouhan et al., licensed to EAI. This is an open access article distributed under the terms of the Creative Commons Attribution licence (http://creativecommons.org/licenses/by/3.0/), which permits unlimited use, distribution and reproduction in any medium so long as the original work is properly cited.

doi: 10.4108/eai.13-7-2018.163133

*Corresponding author. Email:dnchouhan1981@gmail.com

\section{Introduction}

Location estimation of smart sensors in various applications like agriculture, military, and smart cities is possible due to intelligent monitoring of homes, hospitals, community centers, manufacturing industries, and offices. Wireless sensors employed in these workplaces should be smart, tiny, low costs, low data rate and able to be self-organized. Wireless Sensor Networks (WSNs) follow IEEE 802.15.4 standard and it is a multi-hop network that communicates at the short distance and finds its place in a variety of applications like agriculture, volcano monitoring, habitat monitoring, battlefield surveillance, body area network, water quality monitoring, and healthcare industries. One of the biggest challenges of the WSNs is to find out the physical location of the sensor node because without location information the above- said applications cannot produce fruitful information [1]. The easiest solution for this problem is to equip the sensor node with the Global
Positioning System (GPS), but due to this sensor node becomes bulky, costly and consumes lots of energy. The GPS-less outdoor localization scheme is proposed and analyzed for the dense networks in [2]. This scheme does not provide an accurate solution for indoor applications, some improvements also have done for this kind of localization scheme.

Every node in the WSNs cannot be equipped with GPS due to above said reasons so other techniques have been employed in which only a few nodes are attached with GPS and only such nodes in the system know its positions accurately and known as anchor nodes or landmarks. Using range-based and range-free methods the location of remaining nodes is estimated with the help of anchor nodes. A survey presented in [3] classified the localization algorithm of WSNs into two broad categories of learningbased and non-learning based algorithms. The Use of the localization algorithm mainly depends on the nature of applications where it is being used. According to that survey, mobility prediction can be a good technique to estimate the position of the sensor nodes. 
Mobility prediction can be done using various methods either by using movement history-based mobility prediction, physical topology based mobility prediction or logical topology based mobility prediction [4]. History-based prediction predicts the future location by analyzing the past record or habitual behavior while physical topology based mobility prediction uses the physical topology of WSNs and therefore, require either GPS or continuous measurement of power between two mobile nodes to know the time till both are connected or not to obtain estimation of nodes location, velocity, and direction. Apart from the introduction, this paper has the following sections literature review, problem formulation, simulation and results, conclusion and references.

\section{Literature review}

$\mathrm{Hu}$ and Evans present a range-free localization algorithm for mobile sensor networks based on the Sequential Monte Carlo method [5]. Initially Monte Carlo method was used in the field of robotic prominently but $\mathrm{Hu}$ and Evans extend it to localization of sensor nodes in WSNs. This Monte Carlo localization (MCL) algorithm was improved by Baggio et al. [6], in which authors used a box localization scheme. The main differences between both the algorithms are the way to use anchor information and the method used for obtaining new samples. Monte Carlo Box (MCB) limits the area to a box of fix dimensions to draw the samples. Yin Lu et al. [7] proposed a new Monte Carlo mobile node localization algorithm using Newton interpolation There are some deficiencies in the MCL algorithm based on rangefinder, which like location probability distribution of the $\mathrm{k}$ moment in the prediction phase only related to the localization of the $\mathrm{k}-1$ moment and the maximum and minimum velocity. And the influences of the motion condition on the movement of the mobile node at $\mathrm{k}$ moment are also not considered before the $\mathrm{k}-1$ moment. In the MCL process of selecting the compelling particles is slow. This paper ascertained a Monte Carlo mobile node localization algorithm using Newton interpolation, which uses the historical trajectory prediction contrivance of the moving node to estimate the speed and direction of the moving node. Particle filter that is optimized by weight of importance to prevent particle collection depletion. Ammar et al. [8] present an extensive survey of state-of-the-art Sequential Monte Carlo (SMC) localization techniques. It presents the SMC algorithms as a thematic taxonomy of localization operation. All the critical characteristics of each existing SMC technique are compared to identify its advantages and disadvantages. The similarities and differences of each scheme are investigated on the basis of significant parameters, namely, localization accuracy, computational cost, communication cost, and the number of samples. Moreover, it discusses the challenges and direction of future research work for each parameter.

$\mathrm{Su}$ and Gerla [9] proposed a mobility prediction method for calculating the link expiration time between two mobile nodes either by using GPS or received power measurement.
More accurate results can be obtained by using GPS while the applications which require less accurate estimation can use received power method to enhance various unicast and multicast routing protocols. Using these predictions, the system reconfigures its route before it gets disconnected. Yuan et al. [10] proposed an RSSI based algorithm for location estimation of sensor nodes in WSNs. One mobile anchor which knows its location roam around in the field among un localized nodes and broadcast its location to unlocalized nodes so on that basis other nodes can calculate its positions. This algorithm may trigger event-based updates frequently.

Yu et al. [11] present a Social-Relationship-based Mobile node Location Prediction scheme using daily routines (SMLPR).This Scheme models application scenarios based on geographic locations and find social relationships of mobile nodes among each other using nodes mobility. After observing the buoyancy of user's behavior resulting from their daily routines, the SMLPR algorithm initially predicts nodes mobility based on the hidden Markov model in different daily periods of time and then redress the prediction results using location coordinate of other nodes which have a strong relationship with the node.

S. Pattnaik and A. Mahapatro [12] investigates the mobility factors in predicting the next location of sensor nodes to guarantee the accuracy of forwarding decisions. The proposed algorithm uses speed, direction, and degree of randomness to predict the mobility path of sensor nodes. Simulation results show that it precisely estimates the next occupied position of the sensor nodes. Zhu et al. [13] proposed a mobility prediction for mobile underwater sensor networks, it is a distributed localization scheme in which anchor nodes are self-localized and perform mobility prediction. Anchors act as a reference node to non-localized nodes. It employs a modified covariance algorithm to predict mobility pattern parameters.

\section{Mobility and Network model}

Network topology is determined by the mobility model used in the system. So many mobility models are available like Reference Point Group Mobility (RPGM), in this model, all the sensor nodes are divided into several groups and each group has a central node, it may be a logical center or a group leader node. If the center is the group leader, then, each group is formed by one leader and several members. The group leader's movement decides the mobility behavior of the entire group [14]. freeway, and Manhattan both the models come into the category of mobility model with geographic restriction. In this model, the movement of nodes is bounded by streets, freeways or any other obstacles. The destination of each motion phase is randomly selected, so a certain amount of randomness still exists for this model. Basically, in these graph-based mobility models, the nodes are moving in a pseudo-random manner on the pathways [15]. It is observed that the mobility models may have different properties and exhibit various mobility characteristics. As a result of this, these mobility models 
behave differently and influence location estimation techniques in different ways. This paper has used a random waypoint mobility model [16] in which each node can change its speed in each time segment before it reaches its destination. Pause time is set to 0 and the minimum node speed is set to 0.1 to avoid speed decay. Average node speed is half of the maximum speed, and it is expressed as a multiple of the communication radius of the nodes.

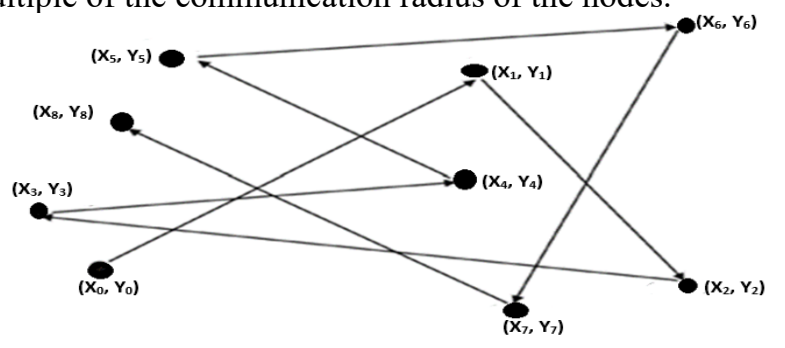

\section{Figure 1. Path Traced by Mobile Nodes in Random Waypoint Model}

The implementation part of the random waypoint mobility model is executed in the following manner as the simulation starts, every mobile node randomly selects one location in the simulation field as the final destination. Then after with constant velocity chosen uniformly and randomly from $[0, \mathrm{~V}]$, It travels towards this destination where the parameter $\mathrm{V}$ is the maximum permissible velocity for every mobile node. The velocity and direction of every node are selected independently of other nodes. After reaching the destination, the node stops for a duration, which is labelled as the 'pause time' parameter. If pause time is zero $(\mathrm{T}=0)$, this leads to continuous mobility. After the pause time, the mobile node again chooses another random destination in the simulation field and moves towards it. This process remains to continue until the simulation period ends [17].

The system under investigation has $\mathrm{N}$ random anchors and $\mathrm{n}$ sensor nodes out of which most of them are mobile and few are stationary. Each of the nodes occupies a position $\left(\mathrm{x}_{\mathrm{i}}, \mathrm{y}_{\mathrm{i}}\right)$ in a fixed area $(1 \times 1 \mathrm{~m} 2)$. This system has a uniform speed and direction of every mobile node, it does not allow the abrupt change in velocity and direction. The Communication radius of every node is R. Every node independently moves in the system and obeys a modified random waypoint mobility model. The velocity of mobile nodes considered in this system under investigation is high.

\section{Link Expiration Mobility Prediction Localization Algorithm (LEMPL)}

The movement of a mobile node in WSNs is not completely random but it moves in some predictable fashion according to the mobility model used. It moves in a predicted manner so the future state of that node in the network under investigation can also be predicted. Most of the localization algorithms do not bother about the total number of packet delivered to its destination successfully sent by the node in the WSNs. This paper incorporates the concept of link expiration with the mobility prediction algorithm. As the expiration time of the link between the anchor and the mobile node is known so before disconnection the mobile node reconfigures its path in the WSNs and helps to deliver the more successful data packets without generating excessive control overhead. The link expiration time can be calculated as in [9], where $\left(\mathrm{x}_{\mathrm{i}}, \mathrm{y}_{\mathrm{i}}\right)$ and $\left(\mathrm{x}_{\mathrm{j}}, \mathrm{y}_{\mathrm{j}}\right)$ are the positions of nodes $i$ and $j$, respectively. And $v_{i}$ and $v_{j}$ be the speeds, moving directions of nodes $i$ and $j$ is $\theta_{i}$ and $\theta_{j}$ respectively, the transmission range of the nodes is $\mathrm{R}$. Then, the amount of time $\mathrm{T}$ the mobile nodes $\mathrm{i}$ and $\mathrm{j}$ will stay connected is given by:

$$
\begin{aligned}
& T=\frac{-(a b+c d)+\sqrt{\left(a^{2}+c^{2}\right) R^{2}-(a d-c b)^{2}}}{\left(a^{2}+c^{2}\right)} \ldots \ldots \ldots \ldots . . .(1) \\
& \text { Where } a=v_{i} \cos \theta_{i}-v_{j} \cos \theta_{j} \ldots \ldots \ldots \ldots \ldots \ldots \text { (2) }
\end{aligned}
$$

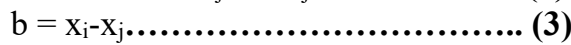

$$
\begin{aligned}
& \mathrm{c}=\mathrm{v}_{\mathrm{i}} \sin \theta_{\mathrm{i}}-\mathrm{v}_{\mathrm{j}} \sin \theta_{\mathrm{j}} \\
& \mathrm{d}=\mathrm{y}_{\mathrm{i}}-\mathrm{y}_{\mathrm{j}}
\end{aligned}
$$

In the above equation, the link time will be very much accurate if the position of the nodes is estimated correctly. The hello message transmitted from the anchor contains anchor id and location of the anchor. The node which receives the hello message that is in the range of that anchor. Initially, the velocity and direction of movement of that node are calculated and using these two parameters and the future position of that node get predicted. This predicted state is compared with the actual state and the difference between both the states is considered as localization error.

\subsection{Link availability estimation}

The link availability estimation is defined as the probability of an active link between anchor node to the mobile node or it can be the probability of an active link between two mobile nodes also at time $t+T$ given that there is an active link between them at time $t$. The link between the node may fail one or more times between time interval $\mathrm{t}$ and $\mathrm{t}+\mathrm{T}$.

Whenever a link fails, then reconfiguration of the path should immediately take place rather than waiting for the lost link to become available again. The link availability criterion is useful for mobile nodes to choose more trustworthy neighbors to form a more stable group. Given an estimation $T$ of the expiration time for an active link which is continuously available $\{v, u\}$ between two nodes $v$ and $u$ at time $t$ is calculated by $\mathrm{Su}$ and Gerla [9] using the link expiration time algorithm, the availability of active link $\{v, u\}$ is defined as $L(T)$ and given as

$L(T)=\operatorname{Pr} \quad$ continuous link of $\{v, u\}$ from time $t$ to time $t+T\}$.(6)

This shows the probability for the link $\{v, u\}$ which will continuously available for duration $t$ to time $t+T$. $L(T)$ can be further divided into two parts, $L_{I}(T)$ when $\{u, v\}$ mobile nodes have the constant speeds and unchanged moving 
directions for time duration time $t$ to time $t+T$, and the link availability $L_{2}(T)$ for the variable speeds and direction of the mobile nodes.

That is, $L(T)=L_{1}(T)+L_{2}(T) \ldots \ldots \ldots \ldots \ldots \ldots \ldots$ (7)

Assuming that the mobility epoch is exponentially distributed with mean $\lambda^{-1}$, and the exponential distribution is 'memoryless', then $L_{1}(T)$ is given by:

$L_{1}(T)=[1-E(T)]^{2}=e^{-2 \lambda T}$

An exact estimation of $L_{2}(T)$ is difficulties due to abrupt changes in speed and direction of the mobile nodes. The approximate prediction $L_{\min }(T)$ of link availability $L_{2}(T)$ is given by Jiang et al.[18]

$L_{\min }(T)=\left(1-e^{-2 \lambda T}\right) / 2 \lambda T+\lambda T e^{-2 \lambda T / 2} \ldots$

The LEMPL is executed as the following steps below

1. Define the field $\left(1 \times 1 \mathrm{~m}^{2}\right)$

2. Deploy the mobile sensors (n)

3. Deploy the anchors $(\mathrm{N})$

4. Send the hello message and search for non-localised nodes in the neighborhood

5. Calculate the velocity of the nodes

$$
\begin{array}{r}
\nu=\frac{\sqrt{\left(x_{i-}-x_{j}\right)^{2}+\left(y_{i-} y_{j}\right)^{2}}}{t} \\
\varnothing=\cos ^{-1} \frac{x_{i-x_{j}}}{\sqrt{\left(x_{i}-x_{j}\right)^{2}+\left(y_{i-} y_{j}\right)^{2}}}
\end{array}
$$

6. Calculate the new position

$$
x_{i+1}=x_{i}+v t \cos \phi
$$

$$
y_{i}+1=y_{0}+v t \sin \emptyset
$$

7. Update the new position (average velocity, direction, and new location)

\section{Calculate the $\mathrm{T}$}

9. If $\mathrm{t}>=\mathrm{T}$ then reconfigure the path

10. Repeat till maximum number of attempts or all nodes get localized

\section{End}

\section{Simulation Results}

The proposed algorithm is implemented in the WSN localization simulator and compared with the SMCL algorithm. Both algorithms are compared over the few parameters, and the conclusion has been drawn. The fix parameters for the network are as shown in table 1. Network parameters are chosen as best suited for the algorithm from
[19]. The number of anchors is selected from a wide range of 10 to 30 to observe the effect on localization error and communication cost. The sensor model is selected is MICA2 which is the most successful product of the Berkeley motes family. The MICA2 platform is equipped with an Atmel ATmega128L and has a CC1000 transceiver, to interface sensor A 51-pin expansion connector is available too. Microcontrollers are incorporating into base band processing and handling medium. An operating system like TinyOS which is an event-driven real-time operating system has been implemented to specifically manage the concurrency and fulfill resource management needs of wireless sensor nodes [20].

\section{Table 1. Network Parameters}

\begin{tabular}{|c|c|}
\hline \multicolumn{2}{|c|}{ Network Parameters } \\
\hline $\begin{array}{c}\text { Number of random } \\
\text { anchors }\end{array}$ & $10,15,20,25,30$ \\
\hline $\begin{array}{c}\text { Number of mobile } \\
\text { sensors }\end{array}$ & 100 \\
\hline $\begin{array}{c}\text { Number of static } \\
\text { sensors }\end{array}$ & Mica2 \\
\hline Sensor model & Two ray ground \\
\hline Propagation model & 1000 Joule \\
\hline Initial energy & 150 Sec. \\
\hline Simulation time & $50 \mathrm{~m}$ \\
\hline Sensors Radio range & \\
\hline
\end{tabular}

The comparison of localization errors between both the algorithms has been made when the number of anchor nodes present is 10. Localization accuracy of the event generated is the most important parameter of WSNs. A fair level of location accuracy can help decision-makers to identify the accurate location and enable the user to take requires measures in the application. Localization accuracy is the variance between coordinates a real location and an estimated location obtained from investigating the scheme. 


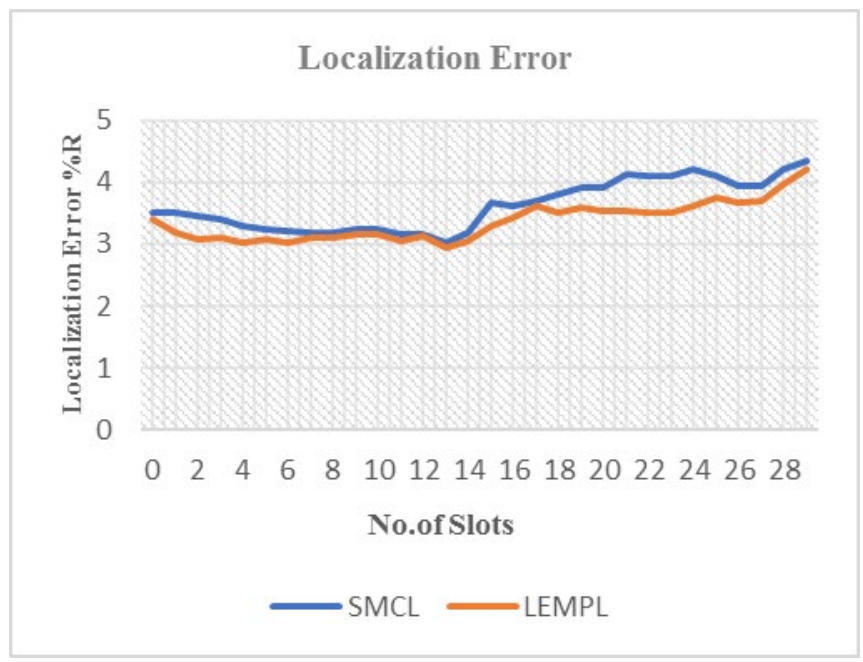

Figure 2. Localization Error (Mobile Nodes $n=100$, Anchor Nodes $\mathrm{N}=10$ )

Localization error is calculated as per slot and SMCL takes the constant number of valid samples while LEMPL uses the variable number of different samples according to need and performs better at both low and high number of nodes with a sufficient number of anchors. Communication cost is determined by the total number of messages broadcasted during the location estimation process. The number of messages is affected by the number of anchor nodes and normal nodes used in the localization process. The size of the broadcasted message also affects communication costs. But at the same time due to fewer packets per slot, the communication cost of SMCL is lesser than LEMPL.

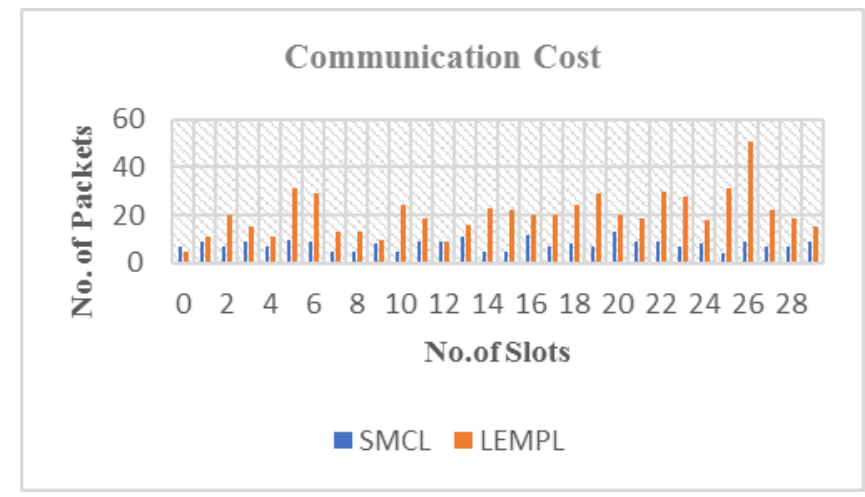

Figure 3. Communication Cost (Mobile Nodes $n=100$, Anchor Nodes $\mathrm{N}=10$ )
The main difference between both the algorithms is lying in the number of valid samples taken, and it is fixed for the SMCL while LEMPL is taking a varying number of valid samples, which results in less communication processing and the saving of energy in wireless sensor nodes, ultimately enhance the lifetime of the sensor node or lifespan of the network.

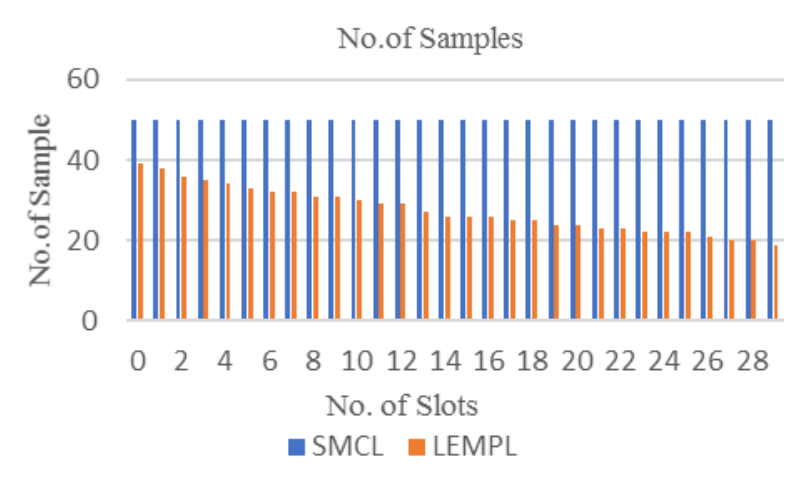

Figure 4. Number of Samples taken to Complete localization (Mobile Nodes $n=100$, Anchor Nodes $\mathrm{N}=10$ )

The number of anchor nodes in the WSNs has direct relations with the localization error but in random deployment, it is not always true. Sometimes if a node is placed in a connectivity hole or at a patch where it is out of network coverage then there is an adverse effect on localization error but as results obtained in general the increase of the number of nodes results in less localization error.

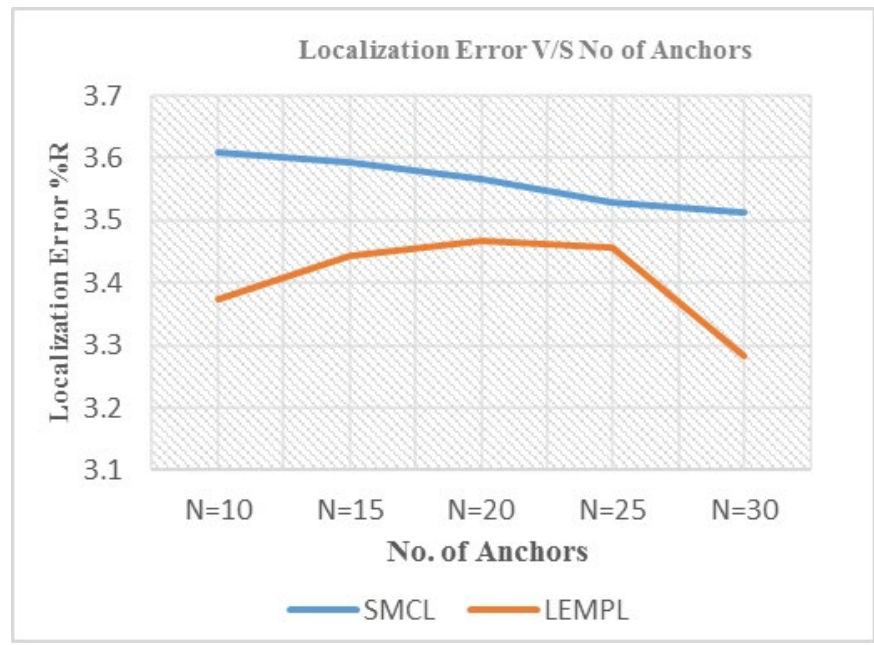

Figure 5. Effect of Increase in Anchor density over Localization Error 


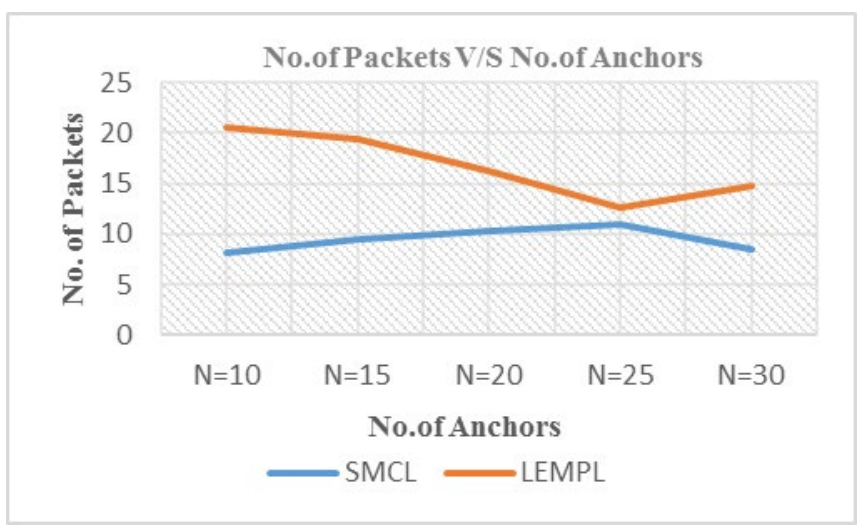

Figure 6. Effect of Increase in Anchor density over Localization Error

With SMCL the increase in the number of anchors hardly plays any role in communication cost, Although LEMPL has high communication costs, and it comes down as the number of anchor node increases.

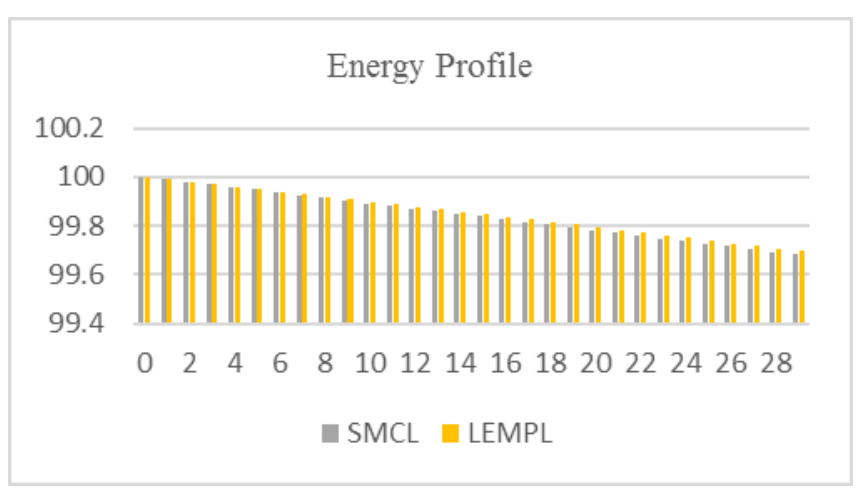

Figure 7. Energy Profile of Sensor Nodes after 150 Sec of Simulation time

As discussed above the varying number of fewer samples for LEMPL results in saving energy too. It is observed from an initial 1000 joule energy in the $150 \mathrm{sec}$. simulation time the LEMPL consumes less energy and its residual energy percent is more than SMCL. The elapsed time of both the algorithms with the varying number of anchor nodes is as shown in table 2 .
Table 2. Elapsed Time

\begin{tabular}{|c|c|c|}
\hline $\mathrm{N}$ & $\mathrm{MCL}$ & LEMPL \\
\hline 10 & $00: 23.687$ & $00: 20.735$ \\
\hline 15 & $00: 24.250$ & $00: 21.906$ \\
\hline 20 & $00: 24.437$ & $00: 21.110$ \\
\hline 25 & $00: 26.687$ & $00: 23.328$ \\
\hline 30 & $00: 27.828$ & $00: 26.953$ \\
\hline
\end{tabular}

As the number of anchor nodes increases the elapsed time both the algorithms increases because it increases the total number of connections available in the networks .but after a certain instant it starts to decrease also.

\section{Conclusion}

Most of the based range-free schemes are used in indoor applications and has low localization accuracy and low communication cost. Due to fewer packets and low message sizes, these are not suitable for outdoor applications, But LEMPL is having better localization accuracy $(6.38 \%$ to $6.55 \%$ better on different anchor density) hence estimating the position of the wireless sensor nodes precisely. It is having high communication costs $(51.72 \%$ high $)$ means utilizing more packets and bigger message sizes in the localization process so it can be a fair choice to use for outdoor localization too. It consumes less amount of power because it is employing fewer samples per slot (average $58.8 \%$ less) so it increases the lifespan of the sensor node and can be deployed in a dense deployment where the battery replacement of sensor nodes is difficult, particularly when nodes are in remote and hazardous areas. When sensor nodes move a small distance without switching to a new connection its Connectivity information remains the same. This study has few limitations in terms of communication costs, and some location error issues when mobile node speed becomes greater than $150 \mathrm{~km} /$ hour. Future work can be done in this direction.

\section{References}

[1] Akyildiz,I.F.,Su,W.,Sankarasubramaniam,Y. and Cayirci, E.(2002) Wireless sensor networks: a survey.Computer Networks Journal 38(4): 393-422.

[2] Bulusu,N.,Heidemann,J. and Estrin,D.(2000) GPS-less low cost outdoor localization for very small devices. IEEE Personal Commun.,7(5):28-34.

[3] Chouhan,D.N.,Mathur,R.and Dubey,T.(2016) Comparative Analysis of Learning and Non Learning based Localization Algorithms in WSNs.International Journal of Sensors, Wireless Communications and Control 6(1):86-93. 
[4] Gavalas D, Konstantopoulos C, Mamalis B, Pantziou G: Mobility prediction in mobile ad-hoc networks. In Next Generation Mobile Networks and Ubiquitous Computing. Edited by: Pierre S. IGI Global, Hershey; 2011:226-2406.

[5] Hu,L. and Evans,D.(2004) Localization for mobile sensor networks.In Proceedings of Tenth International Conference on Mobile Computing and Networking (MobiCom), Philadelphia 26 September - 1 October 2004 (NY:ACM NY),45-57.

[6] Baggio,A. and Langendoen,K.(2006) Monte-Carlo localization for mobile wireless sensor networks, In Proceeding of the Second International Conference on Mobile Ad-hoc and Sensor Networks (MSN), Hong Kong, China, December 13-15, 2006(NY:Springer),318-328.

[7] Lu, JY. and Wang, C. J (2018) A new Monte Carlo mobile node localization algorithm based on Newton interpolation. Wireless Com Network 2018(1): 161.

[8] Ammar M., Znaid A.,Yamani I. M.,Wahid A., Qabajeh L.K., and Omar A.(2017)Mahdi.Sequential Monte Carlo Localization Methods in Mobile Wireless Sensor Networks: A Review. Journal of Sensors vol. (2017):1-19

[9] Su,W. and Gerla,M.(1999) IPv6 Flow Handoff in Ad-Hoc Wireless Networks Using Mobility Prediction. In Proceeding of the IEEE GLOBECOM, Rio de Janeiro, Brazil,Dec.1999, (NY:IEEE ) 271-275.

[10] Yuan,Z. ,Baoli,Z.,Fengqi,Y. and Shufeng,N.A(2009) RSSI based localization algorithm using a mobile anchor node for wireless sensor networks. In Proceeding of the International Joint Conference on Computational Sciences and Optimization, Sanya, Hainan Island, China, Apr 24-26, (NY:IEEE)123-126.

[11] Yu, R., Xia, X., Liao, S., \& Wang, X. (2015). A Location Prediction Algorithm with Daily Routines in Location-Based Participatory Sensing Systems. International Journal of Distributed Sensor

[12] Pattnaik,S. and Mahapatro,A(2015) Energy aware mobility prediction in wireless sensor networks. In Proceeding of the International Conference on Technologies for Sustainable Development (ICTSD),Mumbai,India,Feb.04-06,(NY:IEEE) $1-5$.
[13] Zhu,G,Jiang,R.,Xie,L. andChen,Y.(2014) A distributed localization scheme based on mobility prediction for underwater wireless sensor networks. In Proceeding of the 26th Chinese Control and Decision Conference (CCDC), Changsha,. China, 31 May-2 June(NY:IEEE),4863-4867.

[14] Hong,X.,Gerla,M.,Pei,G., and C.-C. Chiang(1999) A group mobility model for ad hoc wireless networks, in Proc. ACM Intern. Workshop on Modeling, Analysis, and Simulation of Wireless and Mobile Systems (MSWiM),NY,August 1999, (NY:ACM NY),53-60.

[15] Bai,F., Sadagopan,N. and Helmy,A.(2003) a framework to systematically analyze the impact of mobility on performance of routing protocols for ad hoc networks. in Proceedings of IEEE Information Communications Conference (INFOCOM), San Francisco,30 March-3 April 2003 (NY:IEEE ) 85-91.

[16] Broch,J.,Maltz,D.A., Johnson,D.B,.Hu,Y.C. and Jetcheva,J. (1998) A performance comparison of multi-hop wireless ad hoc network routing protocols, in Proceedings of the Fourth Annual ACM/IEEE International Conference on Mobile Computing and Networking(Mobicom98), Dallas , October 1998. (NY:ACM NY),85-97.

[17] Breslau,L.,Estrin,D.,Fall,K.,Floyd,S.,Heidemann,J.,Helmy,A. Huang,P, McCanne,S, Varadhan,K,Xu,Y. and Yu,H.(2000) Advances in network simulation, IEEE Computer 33(5):5967.

[18] Jiang, S., He, D. \& Rao, J., (2001). A Prediction-based Link Availability Estimation for Mobile Ad Hoc Networks. in Proceedings of the IEEE INFOCOM, Anchorage, Alaska, USA, April 2001 (NY:IEEE),1745-1752.

[19] WSN-wireless Sensor Network Localization Simulator-Code Project. Retrieved from: http://www.codeproject.com/ Articles/ 606364/Wireless- Sensor-Network- Localization Simulator-v1.2,feb.2019.

[20] A.P.Chandrakasan (2000),An Architecure for a Power-Aware Distributed Microsensor Nodes, In IEEE workshop on Signal Processing Systems (SiPS"00), Lafayette, LA, Oct.2000 (NY:IEEE),176-187 